\title{
Prognosis and Risk Stratification in Young Papillary Thyroid Carcinoma Patients
}

\author{
Sheng-Fong KUO, TZu-Chieh CHAO*, Chuen HSUEH**, Wen-Yu CHUANG**, Chung-HAN YANG***, \\ JEN-DER LIN ${ }^{\#}$ \\ Division of Endocrinology and Metabolism, Department of Internal Medicine, Keelung Chang Gung Memorial Hospital, Chang \\ Gung University College of Medicine, Taiwan, 204 \\ * Department of General Surgery, Chang Gung Memorial Hospital, Taoyuan, Taiwan, 333 \\ **Department of Pathology, Chang Gung Memorial Hospital, Taoyuan, Taiwan, 333 \\ ***Department of Internal Medicine, Chang Gung Memorial Hospital, Keelung, Taiwan, 204 \\ \#Division of Endocrinology and Metabolism, Department of Internal Medicine, Chang Gung Memorial Hospital, Chang Gung Uni- \\ versity College of Medicine, Taoyuan, Taiwan, 333
}

\begin{abstract}
Controversies remain regarding to the therapeutic methods of papillary thyroid cancer (PTC) in young patients. TNM staging and other risk evaluation system are not perfectly applicable for all young PTC patients in view of disease outcome. The aims of this study are to identify the clinical presentations, prognostic factors and risk analysis methods. From January, 1977, to June, 2006, seventy-seven patients with primary PTC younger than 20 years old at Chang Gung Medical Center in Taiwan were enrolled in this retrospective study. The patients were classified as disease-free or nondisease-free according to presence or absence of distant metastases or local recurrence at the end of follow-up. Clinical data of these patients were analyzed and compared. The average follow-up period was 10.3 years. Two patients died of PTC during the follow-up period; one died of brain metastasis, and one died of airway obstruction. Patients undergoing total thyroidectomy, especially those with disease beyond the thyroid, had better outcomes than patients not undergoing total thyroidectomy $(p=0.003)$. Moreover, the DeGroot clinical classification system was a better predictor of prognosis than TNM $(p<0.001$ vs $p=0.007)$. Our results suggest that prognosis for PTC is not worse in younger patients. However, patients who had undergone total thyroidectomy might have a better prognosis. Clinical classification is a good alternative classification system for predicting disease outcome in young PTC patients. Patients with confined intrathyroid lesion $(\leq \mathrm{T} 2, \mathrm{~N} 0, \mathrm{M} 0)$ may be regarded "low risk" PTC patients.
\end{abstract}

Key words: Papillary thyroid cancer, Pediatric oncology, Prognosis, Cancer staging, TNM classification

(Endocrine Journal 55: 269-275, 2008)

THE prognostic factors for papillary thyroid cancer (PTC) in young patients have not been fully elucidated. Many of the experiences are from large series studies of post-Chernobyl pediatric thyroid cancer $[1,2]$.

There were no conclusive agreement concerning about the management of PTC in young patients. Because of the good prognosis in these young patients,

Received: November 16, 2007

Accepted: November 16, 2007

Correspondence to: Dr. Jen-Der LIN, Division of Endocrinology and Metabolism, Chang Gung Memorial Hospital, 5, Fu-Shin St. Kweishan County, Taoyuan Hsien, Taiwan conservative treatment has been suggested to avoid severe complications [3-5]; on the other hand, aggressive treatment has been suggested by other authors $[6,7]$. Many staging methods have been suggested in order to predict the prognosis for a patient with differentiated thyroid cancer, including the Tumor, Node, Metastasis (TNM) system employed by the American Joint Committee on Cancer (AJCC)/International Union Against Cancer (UICC), which is the most widely used staging method [8], the Age, Metastasis, Extent, Size (AMES) method [9], the Metastasis, Age, Completeness of resection, Invasion, Size (MACIS) method developed by the Mayo Clinic [10] and the DeGroot clinical classifi- 
cation [11]. This retrospective study analyzed the clinical features, treatment results, prognostic factors and cancer staging in these patients and proposed a risk stratification method.

\section{Materials and Methods}

\section{Subjects}

The study included seventy-seven of 2,086 papillary thyroid carcinoma patients younger than 20 years old who received treatment and follow-up at Chang Gung Medical Center, a major tertiary-care hospital in Taiwan, from January, 1977, to June, 2006. The study included patients receiving follow-up treatment for more than one year after surgery. Patients who died of thyroid cancer within one year were also included in the analysis. No subjects had a history of radiation exposure. Most, but not all, subjects received primary treatment at this hospital. The majority of the patients underwent total thyroidectomy with central compartment and selective bilateral neck lymph node dissection. In this study, the term total thyroidectomy refers to near total and total thyroidectomy with limited lymph node dissection; non-total thyroidectomy refers to subtotal thyroidectomy, lobectomy or biopsy. After surgery, most of the patients received long-term thyroid hormone replacement. Cancer screening at this facility consisted of a one to five $\mathrm{mCi}$ radioactive ${ }^{131} \mathrm{I}$ whole body scan, chest X-ray, and serum thyroglobulin (Tg) levels, and were performed at six-month intervals. Once metastatic or recurrent lesions were detected, surgical treatment or ${ }^{131} \mathrm{I}$ ablation therapy was suggested. Some physicians favor therapeutic radioiodine scan for initial treatment instead of low dose diagnostic scan. External beam radiation therapy was used in a few cases of advanced local recurrence or distant bony metastasis according to each physician's choice.

Hospital records were reviewed, and the following data were recorded: age, gender, pre-operative ultrasonographic findings, fine needle aspiration cytology, thyroid function, thyroid scan, histopathology, primary tumor size, surgical method, one-month post-operative serum $\mathrm{Tg}$ level, results of post-operative 1 to $5 \mathrm{mCi}$ of ${ }^{131}$ I cancer work up and therapeutic radioiodine scan, accumulation of therapeutic ${ }^{131} \mathrm{I}$ dose, post-operative chest $\mathrm{x}$-ray findings and cancer staging. At the end of follow-up, patients are divided into disease-free or non-disease-free status. Disease-free status indicated no evidence of disease, defined as absence of distant metastases and no local recurrence based on physical and non-invasive examination. Cancer staging was based on the TNM system described in the AJCC Cancer Staging Handbook, Sixth Edition. This study also applied DeGroot clinical classification due to the young age of the subjects because the TNM includes only two stages for patients under 45 years old. Clinical classification I denotes a tumor with single or multiple intrathyroidal foci. Classification II denotes a tumor with cervical lymph node metastases. Classification III denotes a thyroid tumor with an extrathyroid extension including fixed cervical metastases. Classification IV denotes metastatic lesions outside the neck.

\section{Risk definition}

Based on the TNM staging system, $(\leq \mathrm{T} 2, \mathrm{~N} 0, \mathrm{M} 0)$ were classified as "low risk", and (T3 or T4, any N, any M) or (any T, N1 or M1) were classified as "high risk" in this study; that is, any patients with confined intrathyroid PTC with tumor size $4 \mathrm{~cm}$ or smaller were classified as "low risk", patients with tumor size exceeding $4 \mathrm{~cm}$, or with lymph node metastasis, soft tissue involvement or distant metastasis were classified as "high risk".

\section{Statistical analysis}

Data are presented as mean $\pm \mathrm{SD}$; Chi-square and Mann-Whitney test were used to evaluate the statistical significance of the data. A $p$ value less than or equal to 0.05 was considered statistically significant.

\section{Results}

The age of the seventy-seven patients ranged from 3 to 19 years with a mean age of $16.3 \pm 3.5$ years. Fig. 1 shows the age distribution. Only two patients were under 5 years old, and two patients were between 6 and 10 years old. The study included fifty-nine females and eighteen males. Most patients presented with asymptomatic thyroid nodules. One patient died of thyroid cancer within one year and received follow-up for only one month. Therefore, after a follow-up period ranging from 1 month to 27 years, with an average of $10.3 \pm 5.7$ years and a medium of 9.5 years, all 


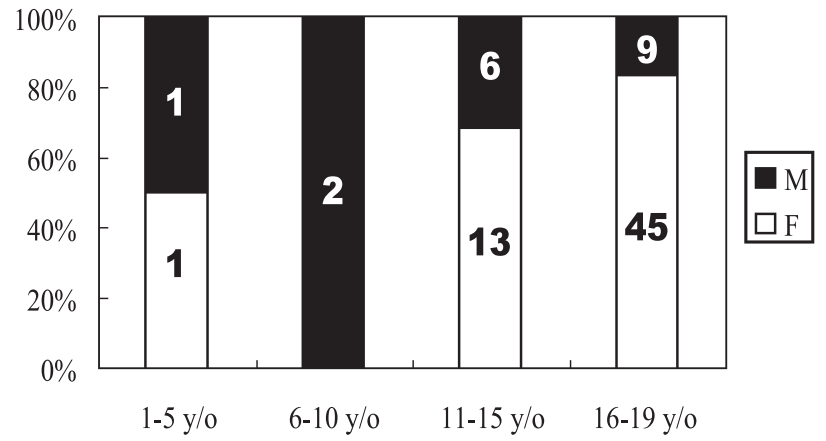

Fig. 1. Age distribution of seventy-seven papillary thyroid carcinoma patients younger than 20 years.

seventy-seven patients survived except for two who died of thyroid cancer.

After surgical intervention with or without radioactive ${ }^{131}$ I treatment, and thyroxine suppressive treatment, sixty-nine of the seventy-seven patients achieved disease-free status at the end of follow-up in June, 2006, including two 16-year-old patients with lung metastasis at diagnosis. Eight patients were in nondisease-free status, including two patients who died of
PTC. Table 1 contains detailed clinical data for the eight non-disease-free patients.

Table 2 compares clinical characteristics between disease-free and non-disease-free patients. Diseasefree patients were older than non-disease-free patients $(p=0.048)$. However, if we divided these patients into patients who are 15 years old or younger, and patients older than 15 years old, there is no statistical significance in disease outcome between them $(p=0.230)$. Of the seventy-seven patients, tumor size was recorded in sixty-five patients. Tumor size did not statistically differ between disease-free and non-disease-free patients $(p=0.205)$. However, some patients in the nondisease-free group had not recorded data for tumor size or exhibited soft tissue invasion making measurement difficult, e.g., patient 1 of table 1 . Fourteen patients had a tumor size exceeding $4 \mathrm{~cm}$. Tumor size larger than $4 \mathrm{~cm}$ was associated with frequent invasion to surrounding soft tissue $(n=5)$ or distant metastasis $(\mathrm{n}=1)$. Fig. 2 depicts Kaplan-Meier disease-free survival curves for these patients in accordance with the definition of risk in this study.

Of the seventy-seven subjects, fifty-three had total

Table 1. Clinical features in eight non-disease-free patients.

\begin{tabular}{|c|c|c|c|c|c|c|c|c|c|}
\hline $\begin{array}{l}\text { Patient } \\
\text { No. }\end{array}$ & Age & Sex & Survival & $\begin{array}{l}\text { Follow-up } \\
\text { period } \\
\text { (years) }\end{array}$ & $\begin{array}{l}\text { Postoperative } \\
{ }^{131} \text { I total dose } \\
\text { (mCi) }\end{array}$ & $\begin{array}{l}\text { DeGroot } \\
\text { class at } \\
\text { diagnosis }\end{array}$ & $\begin{array}{l}\text { TNM } \\
\text { stage }\end{array}$ & $\begin{array}{l}\text { Operative } \\
\text { method }\end{array}$ & $\begin{array}{l}\text { Tumor } \\
\text { size } \\
(\mathrm{cm})\end{array}$ \\
\hline 1 & 10 & M & no & 0.1 & - & III & I & Biopsy only & - \\
\hline 2 & 11 & M & yes & 1.4 & 65.6 & III & I & total thyroidectomy & 4.8 \\
\hline 3 & 13 & $\mathrm{~F}$ & yes & 7.2 & 610 & $\mathrm{IV}^{\mathrm{a}}$ & II & total thyroidectomy & 5.4 \\
\hline 4 & 14 & M & yes & 8.8 & 800 & $\mathrm{IV}^{\mathrm{a}}$ & II & subtotal thyroidectomy & - \\
\hline 5 & 17 & $\mathrm{~F}$ & yes & 5.3 & 60 & III & I & lobectomy & - \\
\hline 6 & 17 & $\mathrm{~F}$ & yes & 12.6 & - & III & I & subtotal thyroidectomy & - \\
\hline 7 & 18 & $\mathrm{~F}$ & no & 1.3 & 150 & $\mathrm{IV}^{\mathrm{b}}$ & II & total thyroidectomy & 2 \\
\hline 8 & 18 & $\mathrm{~F}$ & yes & 3.4 & 275 & III & I & subtotal thyroidectomy & - \\
\hline
\end{tabular}

${ }^{\mathrm{a}}$ Lung metastases; ${ }^{\mathrm{b}}$ Lung, bone and brain metastases.

Table 2. Clinical data for disease-free and non-disease-free subjects at the end of follow-up.

\begin{tabular}{lccc}
\hline & Disease-free $(\mathrm{n}=69)$ & Non-disease-free $(\mathrm{n}=8)$ & $p$ value \\
\hline Sex (female/male) & $54 / 15$ & $5 / 3$ & 0.381 \\
Age & $16.5 \pm 3.3$ & $14.8 \pm 3.2$ & 0.048 \\
Tumor size $(\mathrm{cm})$ & $2.86 \pm 1.28(\mathrm{n}=62)$ & $4.07 \pm 1.81(\mathrm{n}=3)$ & 0.205 \\
Postoperative ${ }^{131}$ I accumulated dose $(\mathrm{mCi})$ & $110.92 \pm 93.33(\mathrm{n}=61)$ & $326.77 \pm 309.02(\mathrm{n}=6)$ & 0.038 \\
Operative method & & & 0.047 \\
$\quad$ Total thyroidectomy & 51 & 3 & 5 \\
$\quad$ Non-total thyroidectomy & 18 & $5.5 \pm 4.5$ \\
Follow-up period (years) & $10.9 \pm 5.5$ & & \\
\hline
\end{tabular}




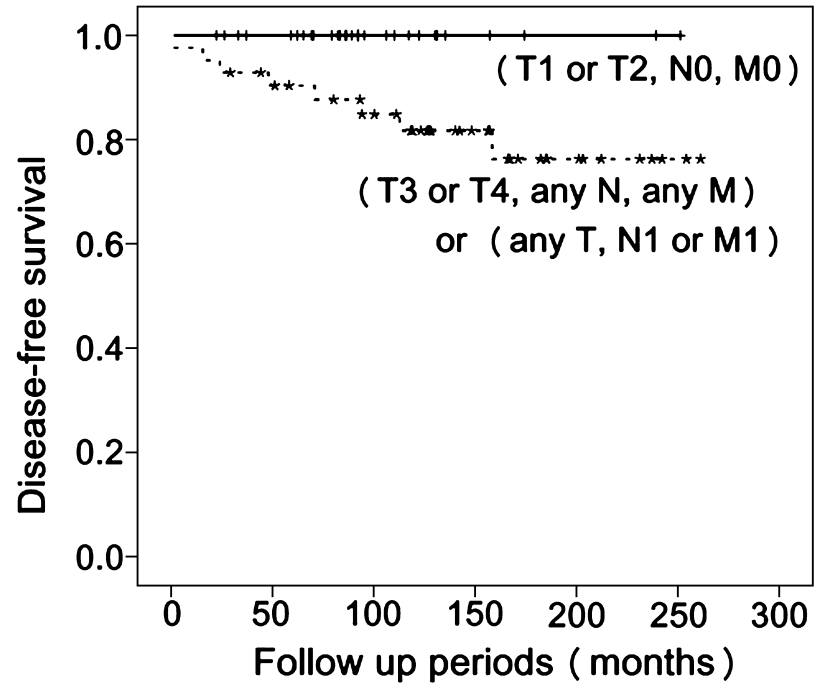

Fig. 2. Kaplan-Meier disease-free survival curves for low-risk patients $(\leq \mathrm{T} 2, \mathrm{~N} 0, \mathrm{M} 0)$ and high-risk patients $[(\mathrm{T} 3$ or $\mathrm{T} 4$, any $\mathrm{N}$, any M) or (any T, N1 or M1)].

thyroidectomy. Twenty-four did not have total thyroidectomy including eighteen with subtotal thyroidectomy, five with lobectomy and one with biopsy only. Operative method and disease outcome significantly differed $(p=0.047)$. Fourteen patients had nodal involvement and sixteen patients had soft tissue invasion in the intraoperative findings. Among the 77 patients, 71 were classical papillary thyroid cancer with features of papillae formation and distinctive nuclear change, including five microcarcinoma and two multicentric papillary thyroid cancer, and six were follicular variant of papillary thyroid cancer. Sixty-seven of the seventyseven patients underwent radioiodine ablation therapy after surgery, including fourteen patients who only received subtotal thyroidectomy or lobectomy for their PTC. The dose of ${ }^{131}$ I required was higher in the nondisease-free patient group than in the disease-free patient group $(p=0.038)$. Six patients underwent external beam radiation therapy.
During the follow-up period, a 10-year-old male (patient 1 in table 1) died of papillary thyroid carcinoma with local invasion to the trachea. The patient expired due to airway obstruction one month after histopathological diagnosis by biopsy with positive Tg staining. Another 18-year-old female (patient 7) died of skull metastasis and brain invasion. During initial surgery for this patient, a $6 \mathrm{~cm}$ thyroid nodule was found, and pathology revealed follicular adenoma. After surgery, the patient did not undergo thyroxine suppressive or radioactive ${ }^{131} \mathrm{I}$ treatment. Lung and multiple bony metastases were found six years after operation. The patient then received surgical treatment for skull and femoral bone metastases. The final histopathological diagnosis of the metastatic cancer and thyroid gland itself was papillary thyroid carcinoma. Despite further treatment with external bean radiotherapy and radioactive ${ }^{131} \mathrm{I}$, the patient died of brain edema 1.3 years after diagnosis.

Table 3 lists the distribution of patients according to TNM staging and clinical classification. Sixteen patients were classified as clinical class III with direct invasion to soft tissue, and five patients were clinical class IV, including four lung metastasis patients and one bone and brain metastasis patient. At the end of follow-up, two patients in clinical classes III and IV, respectively, were died of thyroid cancer. None in clinical class I or II died of thyroid disease. Additionally, all non-disease-free patients were clinical class III or IV, and none were class I or II. Disease-free and nondisease-free status in these patients revealed a closer correlation with clinical classification than with TNM stage, although the difference was not statistically significant $(p<0.001$ vs $p=0.007)$. Fig. 3 presents Kaplan-Meier disease-free survival curves for the seventy-seven patients based on the two staging systems. Patients in clinical classification I \& II were truly disease-free at the end of follow-up, while patients categorized as TNM stage I actually included patients

Table 3. Comparison of two different staging systems and disease outcome.

\begin{tabular}{|c|c|c|c|c|c|c|c|}
\hline & & I & II & III & IV & Total & $p$ value \\
\hline \multirow[t]{3}{*}{ DeGroot classification } & Disease-free & 42 & 14 & 11 & 2 & 69 & $<0.001$ \\
\hline & Non-disease-free & 0 & 0 & 5 & 3 & 8 & \\
\hline & Total & 42 & 14 & 16 & 5 & 77 & \\
\hline \multirow[t]{3}{*}{ TNM stage } & Disease-free & 67 & 2 & & & 69 & 0.007 \\
\hline & Non-disease-free & 5 & 3 & & & 8 & \\
\hline & Total & 72 & 5 & & & 77 & \\
\hline
\end{tabular}




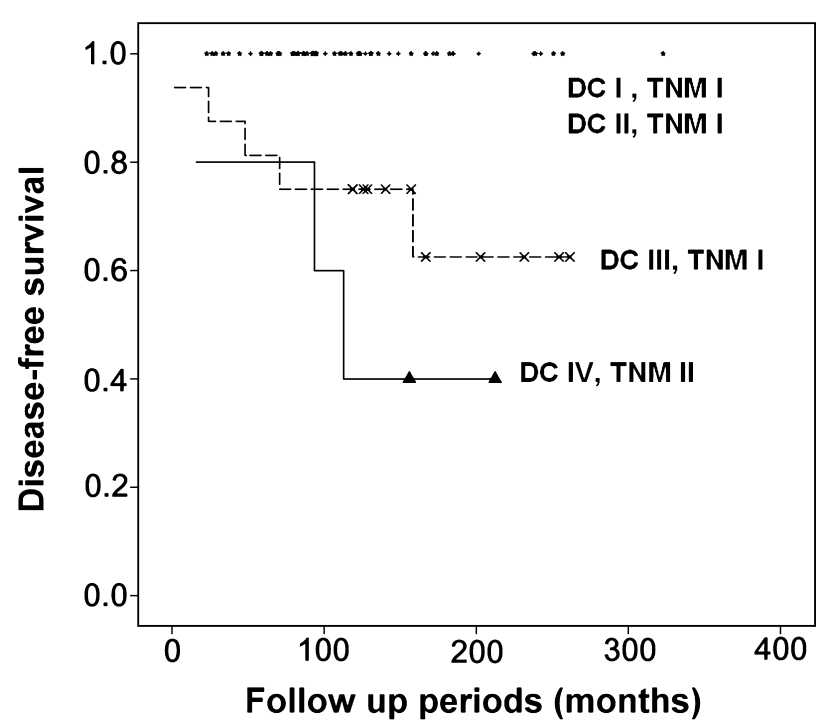

Fig. 3. Kaplan-Meier disease-free survival curves for all seventyseven patients according to DeGroot clinical classification and TNM staging. DC, DeGroot Classification.

in clinical classification I, II and III. Therefore, the prognostic value of the DeGroot clinical classification system was better than the TNM system.

Finally, disease outcome and operative method was analyzed in patients with lymph node metastases, extrathyroid extension and distant metastases (Clinical classifications II-IV). Total thyroidectomy was apparently associated with improved disease outcome in these patients $(p=0.003)$.

\section{Discussion}

Papillary thyroid carcinoma is not common in young patients. Gatta et al. reported that sporadic PTC constitutes only 0.57 percent of all malignancies in children under 15 years old in Europe [12]. This retrospective study in a northern Taiwan hospital was designed to minimize institutional or regional bias. Only fourteen patients in this study were under 15 years old. With age getting older, female gradually predominate indicating that estrogen plays a role in the pathogenesis of PTC $[13,14]$. The objective of PTC treatment in this study was to achieve a disease-free status, i.e., eradication and recurrence-free survival. Disease-free status is clearly related to improved quality of life in children.

Previous reports have demonstrated an excellent prognosis in young PTC patients [15-17]. Only two patients in the current study died of local cancer invasion or distant metastases. Young age is a favorable prognostic factor in patients with well-differentiated thyroid cancer [9, 10, 18, 19]. However, among children, younger patients have been shown to have a poorer prognosis than those diagnosed later in childhood or adolescence [20,21]. Younger age revealed no association with improved prognosis in young PTC patients in the study as well. However, disease outcome did not statistically differ between patients 15 years old or younger and patients older than 15 years old.

Tumor size in well-differentiated thyroid cancer clearly correlates with distant metastasis and survival rate $[9,10,22]$. However, the present results could not confirm an association between increased tumor size and non-disease-free status due to the limited number of non-disease-free patients. Clinically, the tumor size may not be measurable, as in the case of a 10-year-old male in non-disease-free status with cancer invading to adjacent soft tissue and causing large airway obstructions which make the tumor size difficult to measure. Soft tissue invasion is an important factor for prognosis of papillary thyroid carcinoma in young subjects.

Though optimal surgical treatment in young PTC patients is still a matter of debate, total thyroidectomy is regarded as an appropriate treatment strategy $[2,6$, 23]. Total thyroidectomy patients in this study had a better prognosis than those who did not undergo total thyroidectomy. Specifically, total thyroidectomy is most beneficial for patients in clinical classes II, III and IV. Thus, more aggressive surgical intervention should be considered in young PTC patients, especially in those with disease outside the thyroid gland. The majority of patients underwent subsequent radioiodine ablation after surgery, including fourteen patients who only received subtotal thyroidectomy or lobectomy for their PTC. Radioiodine ablation may be feasible and efficacious for managing differentiated thyroid cancer in patients who have undergone less than total thyroidectomy [24-26].

In this study, current TNM staging was not perfectly applicable in all young papillary thyroid cancer patients. Although the difference was not statistically significant, the clinical classification was superior to TNM in predicting response to treatment, because all non-disease-free patients were classified as clinical class III or IV and none as class I or II. In contrast, five patients classified as TNM stage I were actually in nondisease-free status, including one patient who died from 
airway obstruction. Assigning all patients younger than 45 years old to one TNM stage regardless of local tumor invasiveness appears insufficiently objective. Although it is not the most widely used prognostic scoring system, DeGroot clinical classification is an excellent alternative for predicting prognosis in young PTC patients. This study indicated patients with intrathyroid lesions (clinical classification I) are likely to be cured, regardless of operative methods. However, tumor size exceeding $4 \mathrm{~cm}$ was associated with nearby tissues invasion outside the thyroid or distant metastasis. The following risk stratification system based on the TNM staging system, sixth edition is proposed for young PTC patients: patients meeting the criteria $(\leq \mathrm{T} 2$, N0, M0) could be regarded as "low risk"; that is, tumor size not more than $4 \mathrm{~cm}$, no soft tissue invasion or lymph node involvement and no distant metastasis. Conversely, patients grouped as (T3 or T4, any N, any $\mathrm{M})$ or (any T, N1or M1) are regarded as "high risk". This simple risk stratification method resembles European Thyroid Association (ETA) guidelines in which differentiated thyroid cancer patients are classified into three risk categories [27]. Additionally, we compared the operative method and ${ }^{131} \mathrm{I}$ accumulated dose between the patients under 20 years old and those over 20 years old from our database to identify if any difference in treatment methods exist between two groups.
There was no statistical difference toward radioiodine dose $(p=0.8589)$ and operative methods $(p=0.0602)$ between the patients under 20 years old $(n=77)$ and those over 20 years old $(n=1878)$ in our hospital.

Though ${ }^{131}$ I therapy has been suggested in pediatric patients postoperatively $[2,6,28]$, little information is available regarding the appropriate frequency and dosage of ${ }^{131}$ I treatment in children and adolescents with PTC and lung metastases. Two of the present patients who had lung metastases both at age 16 received total doses of 350 and $200 \mathrm{mCi}{ }^{131} \mathrm{I}$, respectively. The metastatic lung lesions subsided after the aforementioned ${ }^{131}$ I treatment, and the patients remained disease-free without complications after eight and eighteen years of follow-up, respectively. Therefore, ${ }^{131} \mathrm{I}$ therapy is effective and should be part of successful treatment in young PTC patients with lung metastasis.

In conclusion, the prognosis of PTC is not worse in young patients. Total thyroidectomy is an optimal treatment and should be performed particularly in patients with disease beyond the thyroid. In view of recurrence-free survival, DeGroot clinical classification is a good alternative for predicting disease outcome in young PTC patients. Moreover, in accordance with the TNM staging system, young PTC patients belonging to $(\leq \mathrm{T} 2, \mathrm{~N} 0, \mathrm{M} 0)$ may be regarded as "low risk".

\section{References}

1. Pacini F, Vorontsova T, Demidchik EP, Molinaro E, Agate L, Romei C, Shavrova E, Cherstvoy ED, Ivashkevitch Y, Kuchinskaya E, Schlumberger M, Ronga G, Filesi M, Pinchera A (1997) Post-Chernobyl thyroid carcinoma in Belarus children and adolescents: comparison with naturally occurring thyroid carcinoma in Italy and France. J Clin Endocrinol Metab 82: 3563-3569.

2. Demidchik YE, Demidchik EP, Reiners C, Biko J, Mine M, Saenko VA, Yamashita S (2006) Comprehensive clinical assessment of 740 cases of surgically treated thyroid cancer in children of Belarus. Ann Surg 243: 525-532.

3. La Quaglia MP, Corbally MT, Heller G, Exelby PR, Brennan MF (1988) Recurrence and morbidity in differentiated thyroid carcinoma in children. Surgery 104: 1149-1156.

4. Brink JS, van Heerden JA, McIver B, Salomao DR, Farley DR, Grant CS, Thompson GB, Zimmerman D, Hay ID (2000) Papillary thyroid cancer with pulmo- nary metastases in children: long-term prognosis. Surgery 128: 881-887.

5. Newman KD, Black T, Heller G, Azizkhan RG, Holcomb GW, Sklar C, Vlarnis V, Haase GM, La Quaglia MP (1998) Differentiated thyroid cancer: determinants of disease progression in patients $<21$ years of age at diagnosis. A report from the Surgical Discipline Committee of the Children's Cancer Group. Ann Surg 237: 533-541.

6. Schlumberger M, De Vathaire F, Travagli JP, Vassal G, Lemerle J, Parmentier C, Tubiana M (1987) Differentiated thyroid carcinoma in childhood: long term follow-up of 72 patients. J Clin Endocrinol Metab 65: 1088-1094.

7. Mazzaferri EL, Jhiang SM (1994) Long-term impact of initial surgical and medical therapy on papillary and follicular thyroid cancer. Am J Med 97: 418-428.

8. Sobin LH, Wittekind CH (2002) UICC: TNM classification of malignant tumors. 6th ed. Wiley-Liss, New York: 52-56. 
9. Cady B, Rossi R (1988) An expanded view of riskgroup definition in differentiated thyroid carcinoma. Surgery 104: 947-953.

10. Hay ID, Bergstralh EJ, Goellner JR, Ebersold JR, Grant CS (1993) Predicting outcome in papillary thyroid carcinoma: development of a reliable prognostic scoring system in a cohort of 1779 patients surgically treated at one institution during 1940 through 1989. Surgery 114: 1050-1057.

11. DeGroot LJ, Kaplan EL, McCormick M, Straus FH (1990) Natural history, treatment, and course of papillary thyroid carcinoma. J Clin Endocrinol Metab 71: 414-424.

12. Gatta G, Capocaccia R, Stiller C, Kaatsch P, Berrino F, Terenziani $M$, the EUROCARE working group (2005) Childhood cancer survival trends in Europe: A EUROCARE working group study. J Clin Oncol 23: 3742-3751.

13. Manole D, Schildknecht B, Gosnell B, Adams E, Derwahl M (2001) Estrogen promotes growth of human thyroid tumor cells by different molecular mechanisms. J Clin Endocrinol Metab 86: 1072-1077.

14. Vivacqua A, Bonofiglio D, Albanito L, Madeo A, Rago V, Carpino A, Musti AM, Picard D, Ando S, Maggiolini M (2006) 17 beta-estradiol, genistein, and 4-hydroxytamoxifen induce the proliferation of thyroid cancer cells through the $\mathrm{g}$ protein-coupled receptor GPR30. Mol Pharmacol 70: 1414-1423.

15. Samuel AM, Sharma SM (1991) Differentiated thyroid carcinomas in children and adolescents. Cancer 67: 2186-2190.

16. Shapiro NL, Bhattacharyya N (2005) Population-based outcomes for pediatric thyroid carcinoma. Laryngoscope 115: 337-340.

17. Collini P, Mattavelli F, Pellegrinelli A, Barisella M, Ferrari A, Massimino M (2006) Papillary carcinoma of the thyroid gland of childhood and adolescence: morphologic subtypes, biologic behavior and prognosis: a clinicopathologic study of 42 sporadic cases treated at a single institution during a 30-year period. Am J Surg Pathol 30: 1420-1426.

18. Gilliland FD, Hunt WC, Morris DM, Key CR (1997) Prognostic factors for thyroid carcinoma: a populationbased study of 15,698 cases from the Surveillance, Epidemiology and End Results (SEER) program 19731991. Cancer 79: 564-573.

19. Lin JD, Chao TC, Sun JH, Ho C, Weng HF (2000)
Trends in the clinical characteristics of patients with papillary thyroid carcinoma in Taiwan. Oncology 58: 280-285.

20. Newman KD, Black T, Heller G, Azizkhan RG, Holcomb GW 3rd, Sklar C, Vlamis V, Haase GM, La Quaglia MP (1998) Differentiated thyroid cancer: determinants of disease progression in patients 21 years of age at diagnosis: a report from the Surgical Discipline Committee of the Children's Cancer Group. Ann Surg 227: 533-541.

21. Alessandri AJ, Goddard KJ, Blair GK, Fryer CJ, Schultz KR (2000) Age is the major determinant of recurrence in pediatric differentiated thyroid carcinoma. Med Pediatr Oncol 35: 41-46.

22. Passler C, Scheuba C, Asari R, Kaczirek K, Kaserer K, Niederle B (2005) Importance of tumour size in papillary and follicular thyroid cancer. Br J Surg 92: 184189.

23. Popovtzer A, Shpitzer T, Bahar G, Feinmesser R, Segal K (2006) Thyroid cancer in children: Management and outcome experience of a referral center. Otolaryngol Head Neck Surg 135: 581-584.

24. Lin JD, Kao PF, Chao TC (1998) The effects of radioactive iodine in thyroid remnant ablation and treatment of well differentiated thyroid carcinoma. $\mathrm{Br} J$ Radiol 71: 307-313.

25. Hoyes KP, Owens SE, Millns MM, Allan E (2004) Differentiated thyroid cancer: radioiodine following lobectomy - a clinical feasibility study. Nucl Med Commun 25: 245-251.

26. Randolph GW, Daniels GH (2002) Radioactive iodine lobe ablation as an alternative to completion thyroidectomy for follicular carcinoma of the thyroid. Thyroid 12: 989-996.

27. Pacini F, Schlumberger M, Dralle H, Elisei R, Smit JW, Wiersinga W, the European Thyroid Cancer Taskforce (2006) European consensus for the management of patients with differentiated thyroid carcinoma of the follicular epithelium. Eur $J$ Endocrinol 154: 787-803.

28. Durante C, Haddy N, Baudin E, Leboulleux S, Hartl D, Travagli JP, Caillou B, Ricard M, Lumbroso JD, De Vathaire F, Schlumberger M (2006) Long-term outcome of 444 patients with distant metastases from papillary and follicular thyroid carcinoma: benefits and limits of radioiodine therapy. J Clin Endocrinol Metab 91: 2892-2899. 
